\title{
Ultrasonography of the larynx: Novel use during the SARS-CoV-2 pandemic (Review)
}

\author{
ROMICA CERGAN $^{1}$, MIHAI DUMITRU ${ }^{1}$, DANIELA VRINCEANU ${ }^{2}$, ADRIANANEAGOS $^{3}$, \\ IONUT ISAIA JEICAN $^{4}$ and RADU CONSTANTIN CIULUVICA ${ }^{1}$
}

\author{
${ }^{1}$ Department of Anatomy, 'Carol Davila' University of Medicine and Pharmacy, 050474 Bucharest; ${ }^{2}$ ENT Department, \\ Bucharest Emergency University Hospital, 010271 Bucharest; ${ }^{3}$ ENT Department, 'George Emil Palade' \\ University of Medicine, Pharmacy, Science and Technology of Targu Mures, 540139 Târgu Mureș; \\ ${ }^{4}$ Department of Anatomy, 'Iuliu Hatieganu' University of Medicine and Pharmacy, 400012 Cluj, Romania
}

Received October 12, 2020; Accepted November 10, 2020

DOI: $10.3892 /$ etm.2021.9704

\begin{abstract}
Few articles have been published on the subject of laryngeal ultrasonography. However, considering the increased power and accuracy of ultrasound technology, this imaging modality should be reevaluated. The present review aimed to increase the awareness of fellow specialists regarding the use of this imaging tool in healthcare units that do not benefit from onsite ear, nose and throat (ENT) service. We illustrate the ultrasonographic examination protocol for the larynx along with the relevant anatomic landmarks. We review cases with laryngeal tumoral pathology that underwent ultrasonographic examination for improved management. All findings were confirmed through computerized tomography (CT) and endoscopy performed by the ENT specialist. The ultrasound of the larynx has potential utility in diagnosis (e.g., laryngeal abnormalities, speech and swallowing abnormalities, identification of endotracheal tube placement), treatment (e.g., guidance of percutaneous tracheostomy and cricothyrotomy) and prognosis (e.g., prediction of postextubation stridor and difficult intubation). This imaging modality could be useful in the current SARS-CoV-2 pandemics in reducing the exposure to invasive maneuvers producing aerosol, such as endoscopy.
\end{abstract}

\section{Contents}

1. Introduction

2. Aims of the ultrasonography of the larynx during the SARS-CoV-2 pandemic

3. Anatomical basis

4. Ultrasonographic findings in laryngeal pathology

5. Conclusions

Correspondence to: Dr Daniela Vrinceanu, ENT Department, Bucharest Emergency University Hospital, Aleea Lunguletu 2, 010271 Bucharest, Romania

E-mail: vrinceanudana@yahoo.com

Key words: ultrasound, larynx, ENT, SARS-CoV-2, COVID-19

\section{Introduction}

If one would query international databases using search words such as larynx and ultrasound, they would find 17 free full text articles on the subject of ultrasonography of the larynx. One of the first articles on this subject was published in 1987 by Raghavendra et al (1). They analyzed 41 healthy volunteers, concluding that sonography may prove to be a potentially useful technique for the examination of the vocal cords. An analysis of 229 healthy volunteers by Hu et al (2) revealed that high frequency sonography can quantitatively measure both true and false vocal cords with good reliability and reproducibility. A detailed anatomical study of the upper airway was performed by Singh et al (3), with potential utility in diagnosis (e.g., laryngeal abnormalities, speech and swallowing abnormalities, identification of endotracheal tube placement), treatment [e.g., guidance of percutaneous tracheostomy and cricothyrotomy (4)], and prognosis [e.g., prediction of postextubation stridor (5) and difficult intubation]. We are still at the level of using ultrasound for analyzing healthy volunteers in order to ascertain whether there are gender differences in vocal cord size and that there is no correlation between vocal cord measurements and individual body mass index (BMI) (6). In a preliminary bedside study, sonography was used for diagnosing acute epiglottitis in the emergency department (7). Ultrasound examination proved especially useful in managing post intubation larynx traumas (8). Moreover, real-time high-resolution ultrasonography was used to assess the status of thyroglossal duct cysts by the ENT surgeon firsthand (9). Finally, the utility of sonography for evaluation of clinical T1 and T2 glottic carcinoma has been proven (10).

\section{Aims of the ultrasonography of the larynx during the SARS-CoV-2 pandemic}

The ultrasonography of the larynx is a diagnostic imaging modality that is rather neglected due to the lack of experience and the proximity of ENT departments that can perform endoscopic diagnosis of laryngeal pathology. However, in an emergency setting, a simple visualization of the larynx could be implemented as a quick extension to any FAST-like 


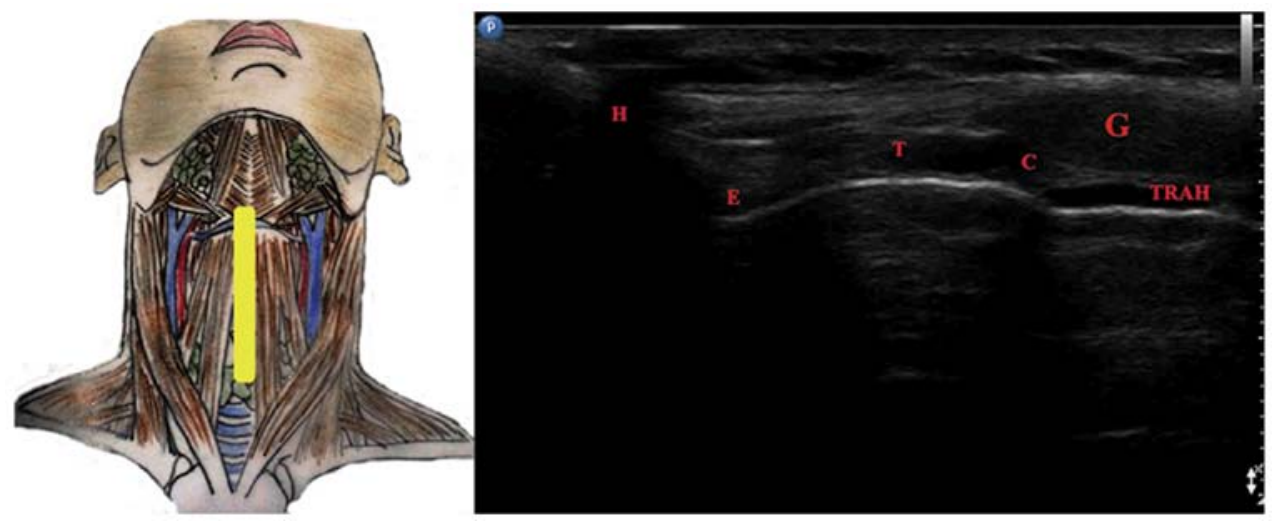

Figure 1. Longitudinal midline view of the larynx. H, hyoid bone; E, epiglottis; T, thyroid cartilage; C, cricoid cartilage; G, thyroid gland isthmus; TRAH, trachea.

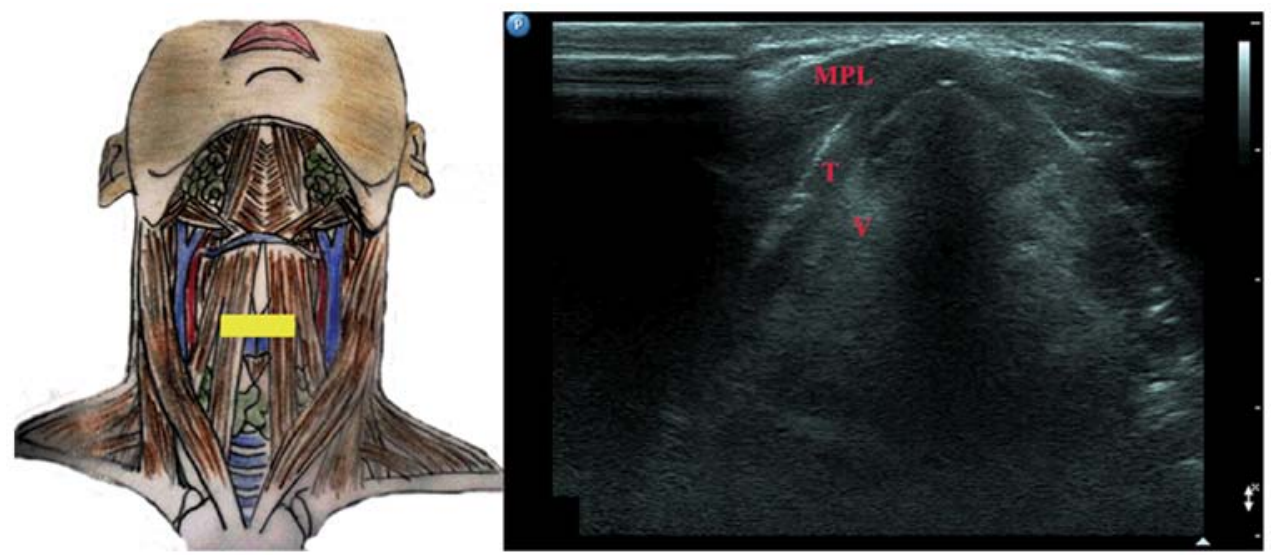

Figure 2. Transverse view at the level of vestibular folds. MPL, anterior muscles of the neck; T, thyroid; V, vestibular folds.

protocol. We hope to increase the awareness of the scientific community regarding this imaging tool that in the future, after a thorough validation and standardization, could lower the associated costs of other investigations. Furthermore, current guidelines encourage reducing the use of aerosol producing procedures, such as laryngeal endoscopy during the SARS-CoV-2 pandemic. Laryngeal ultrasound could lower the risk of ENT specialists in contracting the COVID-19 viral infection.

\section{Anatomical basis}

We reviewed cases with laryngeal pathology that received ultrasound examination. All cases had ultrasonographic findings confirmed by $\mathrm{CT}$ and underwent surgery with subsequent pathology diagnosis. All images presented in this review come from patients admitted to a tertiary ENT department. All patients signed an informed consent form in compliance with the Declaration of Helsinki and current Good Clinical Practice.

We used a Sonoscape S2 machine with a linear probe in order to examine the patients with laryngeal pathology. The patient was examined in a horizontal position with the head in hyperextension. The first step included a longitudinal midline view of the larynx and trachea with direct inspection of the hyoid bone $(\mathrm{H})$, epiglottis $(\mathrm{E})$, thyroid cartilage $(\mathrm{T})$, cricoid cartilage $(\mathrm{C})$, thyroid gland isthmus $(\mathrm{G})$ and trachea (TRAH) (Fig. 1).

Fig. 2 illustrates the vestibular folds in transverse view: MPL, anterior muscles of the neck; T, thyroid cartilage and V, vestibular folds.

The vocal cords are visualized in Fig. 3 in transverse view: MPL, anterior muscles of the neck; T, thyroid cartilage; CV, vocal cords and ARI, arytenoid cartilage.

\section{Ultrasonographic findings in laryngeal pathology}

There are many sonography signs and anatomy landmarks that need to be explained, illustrated, and understood by clinicians before relying on ultrasound diagnosis at the level of the larynx.

Any imaging modality should be valuable in visualizing the extent of the tumor. In a 50-year-old male patient presenting with breathing difficulty and impaired swallowing, ENT exam revealed a tumor comprising the epiglottis and extending into the larynx. The CT showed that the tumor extended from the epiglottis downwards to the level of the cricoid cartilage. The ultrasonographic exam confirmed the complete neoplastic change in the content of the larynx. All these findings were confirmed through total laryngectomy (Figs. 4 and 5).

Fig. 6 comprises serial transverse images of a 70-year-old patient, presenting a pharyngolaryngeal tumor invading the 


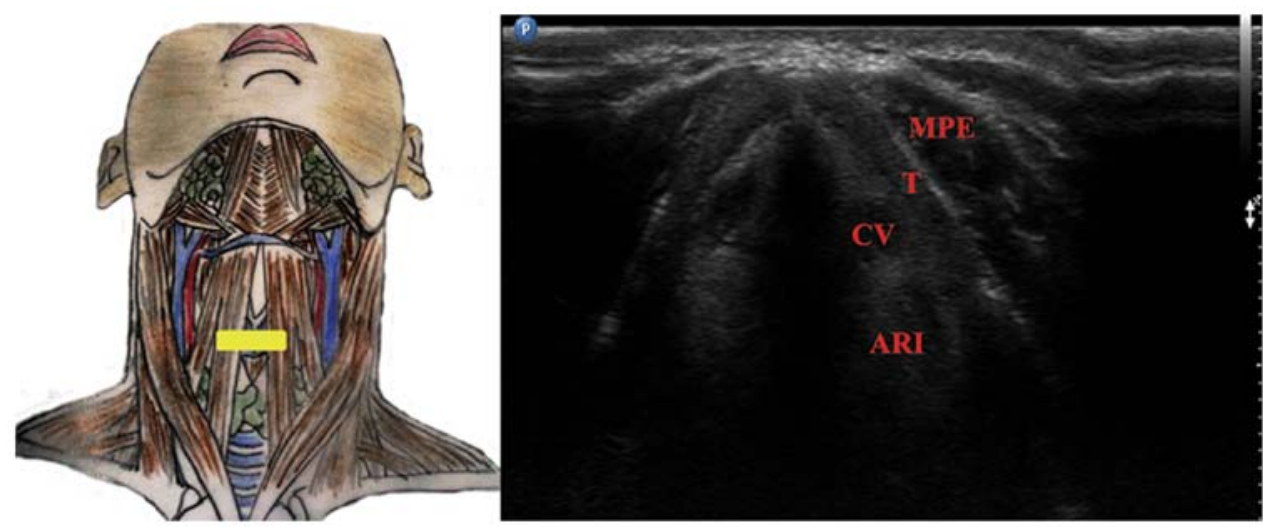

Figure 3. Ultrasonographic view of the vocal cords. MPL, anterior muscles of the neck; T, thyroid cartilage; CV, vocal cords; ARI, arytenoid cartilage.

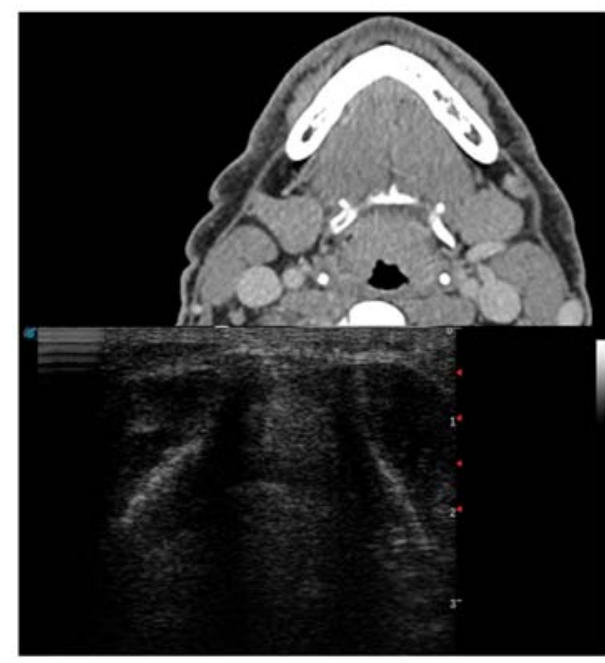

Figure 4. Endolaryngeal tumor computerized tomography (CT) and ultrasound (US) view.

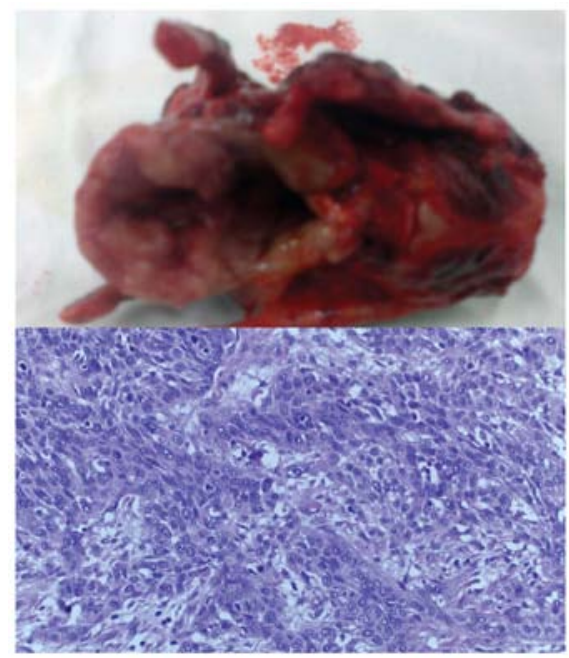

Figure 5. Resection piece and microscopy pathology result, squamous carcinoma, in the same case as shown in Fig. 4. H\&E; magnification, x100. H\&E, hematoxylin and eosin.

base of the tongue, the epiglottis and extending downwards through the vestibular folds until the level of the anterior part

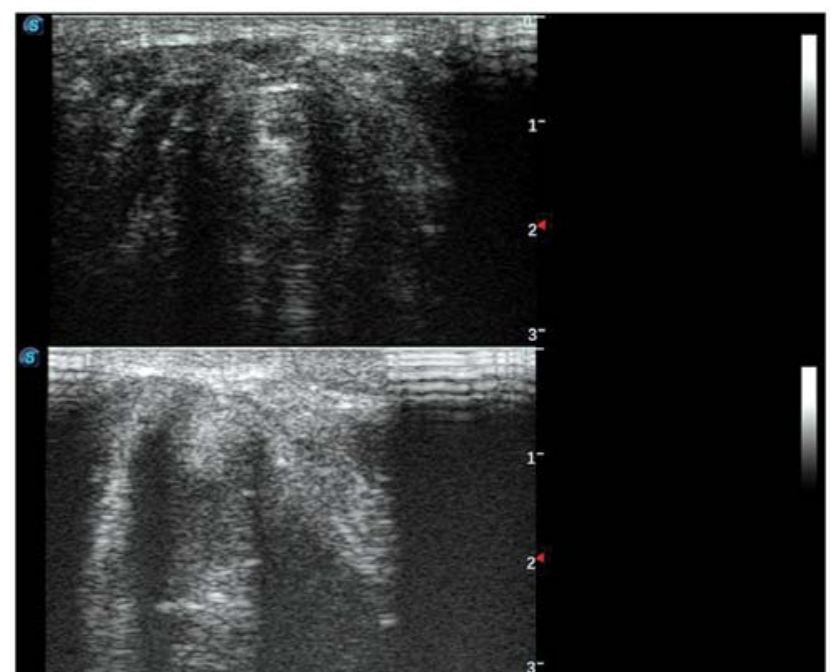

Figure 6. Upper view of the tumor at the base of the tongue and epiglottis, middle view of the tumor at the level of the vestibular folds and lower view of the tumor invading the vocal cord.

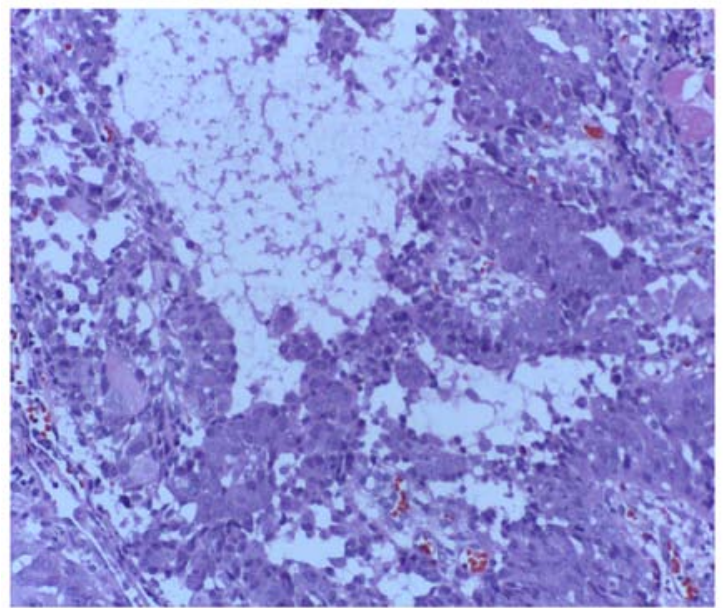

Figure 7. Microscopy pathology result, acantholytic squamous carcinoma, from the same case as shown in Fig. 6. H\&E magnification, x100. H\&E, hematoxylin and eosin.

of the left vocal cord. The pathology result confirmed an acantholytic squamous laryngeal carcinoma (Fig. 7). 


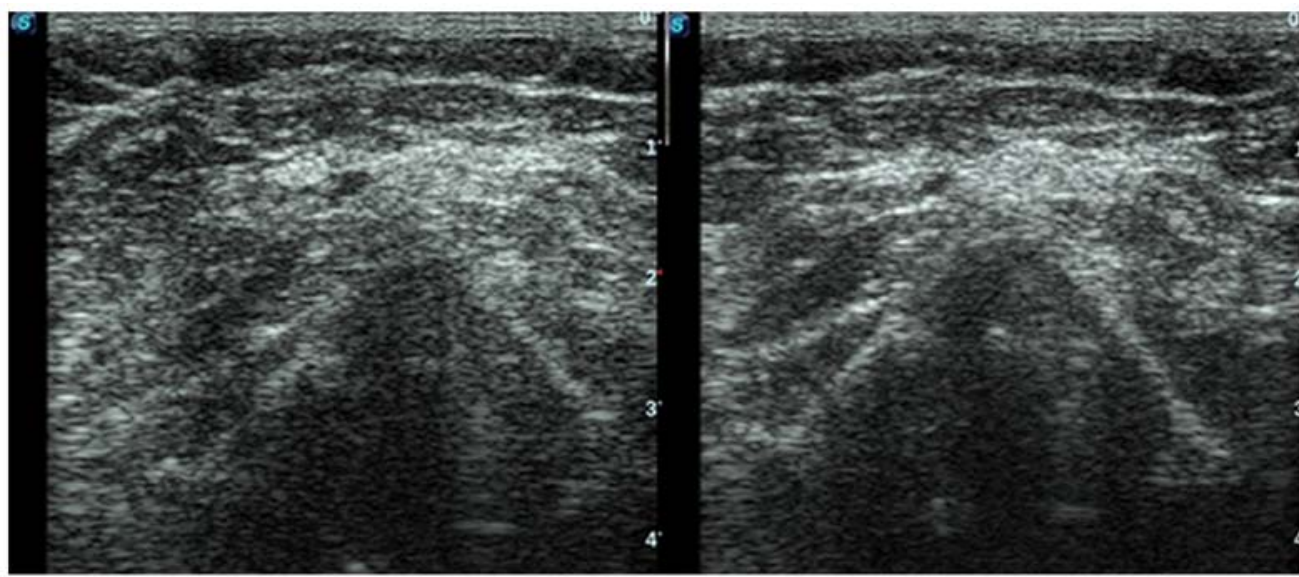

Figure 8. Vocal fold paresis confirmed through ultrasound (US).

Another situation is that of an external mass effect. A 66-year-old woman with a history of thyroid pathology that neglected endocrinology treatment presented with a giant tumor comprising the left thyroid lobe and extending to adjacent structures. CT raised the suspicion of vocal fold paresis. This was confirmed through ultrasonography and endoscopy with subsequent biopsy (Fig. 8). The patient was referred to oncologic palliative care.

Moreover, laryngeal ultrasound has a promising use in trauma departments enabling quick analysis of the motility of the vocal cords (11).

SARS-CoV-2 pandemic changed current diagnosis and treatment protocols worldwide, minimizing the use of aerosol producing maneuvers, such as indirect or direct flexible laryngoscopy, at least until the development of a safe vaccine $(12,13)$.

\section{Conclusions}

The ultrasonographic imaging of the larynx is a rather neglected imaging tool, mainly due to $\mathrm{CT}$ and magnetic resonance imaging (MRI) being accessible. However, ultrasonography is valuable in emergency room settings as an extension of FAST protocols in neck trauma. This review confirmed the fact that ultrasound enables indirect examination of the vocal cords even by doctors of other specialties other than ENT surgeons. Moreover, ultrasound was used in cases where further information was required for the management of the case, minimizing the costs associated with other advanced imaging modalities. Needless to mention the fact that ultrasonography is fast, nonradiating and permits serial real-time examination of the patient. Further studies are needed in order to gather data concerning the sensitivity and specificity of this imaging modality. New guidelines issued during the SARS-CoV-2 pandemic recommend reducing the use of aerosol producing procedures, such as video endoscopy. Laryngeal ultrasound could be used to prevent the exposure of ENT specialists to COVID-19.

\section{Acknowledgements}

Professional editing, linguistic and technical assistance performed by Irina Radu, Individual Service Provider.

\section{Funding}

No funding was received.

\section{Availability of data and material}

All data generated or analyzed during this study are included in this published article.

\section{Authors' contributions}

RC and MD contributed substantially to the conception and design of the study, the acquisition, analysis and interpretation of the data, and were involved in the drafting of the manuscript. DV and AN contributed substantially to the acquisition, analysis and interpretation of the data and were involved in the drafting of the manuscript. RCC and IIJ contributed substantially to the acquisition of the data and were involved in the critical revisions of the manuscript for important intellectual content. All authors agreed to be accountable for all aspects of the work in ensuring that questions related to the accuracy or integrity of any part of the work are appropriately investigated and resolved. All authors read and approved the final version of the manuscript.

\section{Ethics approval and consent to participate}

The information concerning the patients followed the international regulations in accordance with the Declaration of Helsinki.

\section{Patient consent for publication}

Patient informed consent for publication of the data/images associated with the manuscript was obtained. The authors followed the international and national regulations in accordance with the Declaration of Helsinki and all identifying information was removed.

\section{Competing interests}

The authors declare that they have no competing interests. 


\section{References}

1. Raghavendra BN, Horii SC, Reede DL, Rumancik WM, Persky WM and Bergeron T: Sonographic anatomy of the larynx, with particular reference to the vocal cords. J Ultrasound Med 6: 225-230, 1987

2. Hu Q,Zhu SY, Luo F, Gao Y and Yang XY: High-frequency sonographic measurements of true and false vocal cords. J Ultrasound Med 29: 1023-1030, 2010

3. Singh M, Chin KJ, Chan VW, Wong DT, Prasad GA and Yu E: Use of sonography for airway assessment: An observational study. J Ultrasound Med 29: 79-85, 2010.

4. Suzuki A, Iida T, Kunisawa T, Henderson JJ, Fujita S and Iwasaki H: Ultrasound-guided cannula cricothyroidotomy. Anesthesiology 117: 1128, 2012.

5. Ding LW, Wang HC, Wu HD, Chang CJ and Yang PC: Laryngeal ultrasound: A useful method in predicting post-extubation stridor. A pilot study. Eur Respir J 27: 384-389, 2006.

6. Sólyom R, Csiszér I and Neagos A: Tonsillar hypertrophy implications in sleep disorders in adults and children. Rom J Morphol Embryol 55 (2 Suppl): S603-S606, 2014.

7. Ko DR, Chung YE, Parl I, Lee HJ, Park JW, You JS, Chung TN, Park YS, Chung SP and Kim S: Use of bedside sonography for diagnosing acute epiglottitis in the emergency department: A preliminary study. J Ultrasound Med 31: 19-22, 2012.

8. Costache A, Dumitru M, Tweedie D, Sarafoleanu C and Anghel I: Adult cervical lymphangioma-ultrasonography, surgical removal, and pathology results. Case report. Med Ultrason 17: 411-413, 2015.
9. Vrinceanu D, Dumitru M, Cergan R, Anghel AG, Patrascu ET, Sarafoleanu CG and Costache A: Correlations between ultrasonography performed by the ENT specialist and pathologic findings in the management of three cases with thyroglossal duct cyst. Med Ultrason 20: 524-526, 2018.

10. Costache A, Dumitru M, Anghel I, Cergan R, Anghel AG and Sarafoleanu C: Ultrasonographic anatomy of head and neck-a pictorial for the ENT specialist. Med Ultrason 17: 104-108, 2015.

11. Anghel AG, Anghel I, Dumitru M and Soreanu CC: Respiratory and phonatory impairment due to iatrogenic vocal fold paralysis and paresis. A retrospective study of 188 patients. Rom J Leg Med 20: 287-290, 2012.

12. Docea AO, Tsatsakis A, Albulescu D, Cristea O, Zlatian O, Vinceti M, Moschos SA, Tsoukalas D, Goumenou M, Drakoulis N, et al: A new threat from an old enemy: Re-emergence of coronavirus (review). Int J Mol Med 45: 1631-1643, 2020.

13. Calina D, Docea AO, Petrakis D, Egorov AM, Ishmukhametov AA, Gabibov AG, Shtilman MI, Kostoff R, Carvalho F, Vinceti M, et al: Towards effective COVID-19 vaccines: Updates, perspectives and challenges (review). Int J Mol Med 46: 3-16, 2020.

This work is licensed under a Creative Commons Attribution-NonCommercial-NoDerivatives 4.0 International (CC BY-NC-ND 4.0) License. 